Andrew P. McPherson* and Youngmoo E. Kim ${ }^{\dagger}$

*School of Electronic Engineering and Computer Science

Queen Mary, University of London

Mile End Road

London E1 4NS, UK

${ }^{\dagger}$ Department of Electrical and

Computer Engineering

Drexel University

3141 Chestnut St.

Philadelphia, Pennsylvania 19104, USA

andrewm@eecs.qmul.ac.uk, ykim@drexel.edu

http://andrewmcpherson.org,

http://music.ece.drexel.edu

\title{
The Problem of the Second Performer: Building a Community Around an Augmented Piano
}

\begin{abstract}
The design of a digital musical instrument is often informed by the needs of the first performance or composition. Following the initial performances, the designer frequently confronts the question of how to build a larger community of performers and composers around the instrument. Later musicians are likely to approach the instrument on different terms than those involved in the design process, so design decisions that promote a successful first performance will not necessarily translate to broader uptake. This article addresses the process of bringing an existing instrument to a wider musical community, including how musician feedback can be used to refine the instrument's design without compromising its identity. As a case study, the article presents the magnetic resonator piano, an electronically augmented acoustic grand piano that uses electromagnets to induce vibrations in the strings. After initial compositions and performances using the instrument, feedback from composers and performers guided refinements to the design, laying the groundwork for a collaborative project in which six composers wrote pieces for the instrument. The pieces exhibited a striking diversity of style and technique, including instrumental techniques never considered by the designer. The project, which culminated in two concert performances, demonstrates how a new instrument can acquire a community of musicians beyond those initially involved.
\end{abstract}

Composers often speak of the problem of the second performance: Many ensembles commission and perform new works, but fewer offer chances for pieces premiered elsewhere to be heard a second time. In the field of digital musical instrument (DMI) design, we instead encounter the problem of the second performer: Once a new instrument has been built and the first performances have been given, how can the designer establish a continuing role for the instrument in the broader musical community? Very few new instruments have attracted a significant following, with the result that an instrument's designer is often its only performer and composer. Because designing an instrument is time-consuming, and many designers are constantly creating new instruments, even the most dedicated of designers will struggle to establish a musical presence for his or her creations. Sergi Jordà summarizes: "Many new

Computer Music Journal, 36:4, pp. 10-27, Winter 2012 (c) 2013 Massachusetts Institute of Technology. instruments are being invented. Too little striking music is being made with them" (Jordà 2004, p. 326).

This article addresses the stage in DMI design following the creation of the first prototype and the presentation of the first performances. Our primary purpose is not to specify how a DMI should be initially designed or evaluated, a topic covered in many excellent articles (Wanderley and Orio 2002; Jordà 2004; Cook 2009; Paine 2009; Gurevich and Fyans 2011; O'Modhrain 2011). Rather, we examine the process of bringing an instrument to a larger community of musicians, sometimes changing its design in the process. As a case study, we present our recent work with composers and performers using the magnetic resonator piano, an electronically augmented acoustic grand piano which gives the pianist continuous control over the pitch, dynamics, and timbre of every note. The article concludes with general recommendations for DMI designers seeking to establish a continuing musical life for their instruments. 


\section{Piece or Instrument?}

Perry Cook advises DMI designers starting a project to "make a piece, not an instrument or controller" (Cook 2009, p. 218). DMI composer-designers have the unique luxury of being able to build the instrument to suit the needs of the piece, and this is indeed a common practice (Paine 2009). There are very good reasons to take this approach: Simply put, if an instrument is not useful to its creator, it is unlikely to be of use to anyone else.

Jordà describes the instrument creation process as digital lutherie, a mix of science and art (Jordà 2004). When the lutherie process breaks down, it is just as often from lack of artistic planning as from technical failure: Most attendees of electronic music concerts have at some point experienced unsatisfying "knob-twiddling" performances stemming from inadequate musical planning (Schloss 2002). It seems reasonable, then, for DMI design to initially focus on producing the strongest possible musical result rather than the theoretically broadest capabilities.

Musical communities are more easily formed around instruments than pieces, however. The DMI community includes many singular experts on their own creations, which have been carefully tailored to the creator's artistic requirements. Though this is a perfectly acceptable arrangement, engaging other users involves allowing them to assert their own individuality on the instrument. Every common acoustic instrument affords the performer the ability to develop a personal expressive style, and a DMI too closely designed for the needs of a single piece may pose unacceptable constraints on other musicians. In the second stage, Cook rightly argues, DMI designers should consider ways to make the instrument less piece-specific and more generically useful.

\section{Musician-Focused Design}

Consider an alternative approach, taken either from the outset of a project or at the revision stage. Instead of designing for a specific piece, or even for an individual composer, the designer can take a user-centered approach, shaping the instrument to fit the perceived requirements of a community. Literature surveys, musician interviews, and community observation can produce a set of design goals which ideally result in a more generically useful instrument.

One challenge in user-centered DMI design is that there are many potential stakeholders; the performer, the composer, the audience, the designer, and the manufacturer may each have different and sometimes contradictory perspectives on what constitutes a successful instrument $\left(\mathrm{O}^{\prime}\right.$ Modhrain 2011). For example, the easiest way to control a musical process may not be the one that gives the audience the clearest visual indication of cause and effect, the latter being highly important to a convincing performance (Schloss 2002). Moreover, a single individual may at different times occupy different roles. In particular, potential future DMI performers may begin as audience members, and if the instrument does not engage the audience, the opportunity for future collaboration may be lost.

\section{The Pitfall of Over-Determination}

Could user-centered DMI design in fact become counterproductive? Johan Redström argues that the very concept of a "user" is artificial, in that a person only becomes a user when there is a specific object to use (Redström 2006). Attempting to design for a particular set of usage scenarios risks "trapping people in a situation where the use of our designs has been over-determined and where there is not enough space left to act and improvise"(p. 129). Musicians frequently explore and repurpose devices in ways their designers did not anticipate; Redström cites the example of turntable use by DJs, one of many cases of repurposing throughout music history.

Former Apple CEO Steve Jobs, when asked what market research went into the iPad, replied: "None. It's not the consumers' job to know what they want" (New York Times 2011). Similarly, the performer's primary job is to express him- or herself using the instrument at hand. This job does not necessarily extend to imagining hypothetical future capabilities, though some performers also have a talent for this. 
Particularly if we view digital lutherie as a mix of artistry and scientific research, the instrument designer can be well served to follow his or her own artistic intuition instead of trying to respond exclusively to the perceived needs of others.

Our view, which we will examine in more depth in the case of the magnetic resonator piano, is that user feedback is critical to later stages of redesign and community-building; in the first stage, designing for a community of one is sufficient. (This is not to say pre-design user studies should never be conducted, only that the designer's intuition has an important role to play in DMI creation.) To summarize our argument: Just build it, give it to musicians, and learn from what they do. The reason for this partly lies in the difference between how designers and new users explore the capabilities of an instrument.

\section{Affordances and Constraints}

Thor Magnusson examines DMI design from the twin perspectives of affordances and constraints (Magnusson 2010). An affordance can be defined as a system's perceived capacity for a particular action. It is a property of the relationship between human and system: A musical instrument affords certain actions to a human player (dragging a bow across a string, pressing a piano key). The constraints of an instrument are its limitations, which may be obvious or subtle (no pitch bending on the piano, limited polyphony on the violin).

When an instrument is designed around a piece, the designer is likely to consider affordances: The piece will set specific artistic goals, and the designer will find ways for the performer to achieve them. Subsequent performers and composers, however, will encounter a very different situation: The instrument will now be a fixed, complete object for which new music must be created. These musicians will explore the instrument's set of capabilities with the goal of making it serve their own artistic ends. Each musician may arrive at a different, even idiosyncratic interpretation of the instrument's affordances, but all musicians will be bound by essentially the same set of limitations, leading Magnusson to argue that "learning a digital musical instrument is therefore more appropriately described as 'getting a feeling' for the instrument's constraints, rather than engaging with its affordances" (Magnusson 2010, p. 65)

For some DMIs, however (especially augmented instruments building on traditional technique), performer skill is also an implicit constraint: Not all the techniques of an expert performer will be available to a novice. Nicolas Rasamimanana models the performer-instrument relationship as a "space of possibilities" defined by the intersections of instrument acoustics, general bio-mechanical limitations and the skills of an individual performer (Rasamimanana 2012, p. 218). Particularly promising for augmented instrument design is the set of gestures that are bio-mechanically possible and within the performer's skill but that have no traditional acoustic function: In this case, the constraints of the traditional instrument can be relaxed to give the performer a wider expressive space to explore.

Two performer studies on opposite ends of the complexity spectrum support Magnusson's model of constraint exploration. Gurevich, Stapleton, and Marquez-Borbon (2010) asked musicians to develop a performance using an instrument consisting of a single button that played a fixed-frequency tone. The nine participants developed a striking diversity of techniques, and accidental discovery was found to be an important process in developing a personal style. Newton and Marshall (2011) developed a toolkit for performers to create their own augmented instruments. Instead of setting musical goals in advance, participants took an exploratory approach focused on limitations and permutations of the technology.

We observed a similar process of constraint exploration in working with musicians on the magnetic resonator piano. Because constraints play such a crucial role in a new performer's evaluation of an instrument, we argue that building a community around a DMI requires careful attention to constraints, especially to deciding which constraints can be relaxed in later design revisions. We can thus refine our argument above to: "Just build it. Musicians will do something unexpected with it anyway, so learn from what they do." 
Figure 1. Pianist Feifei

Zhang playing the

magnetic resonator piano.

(Photo: Tony Solitro.)

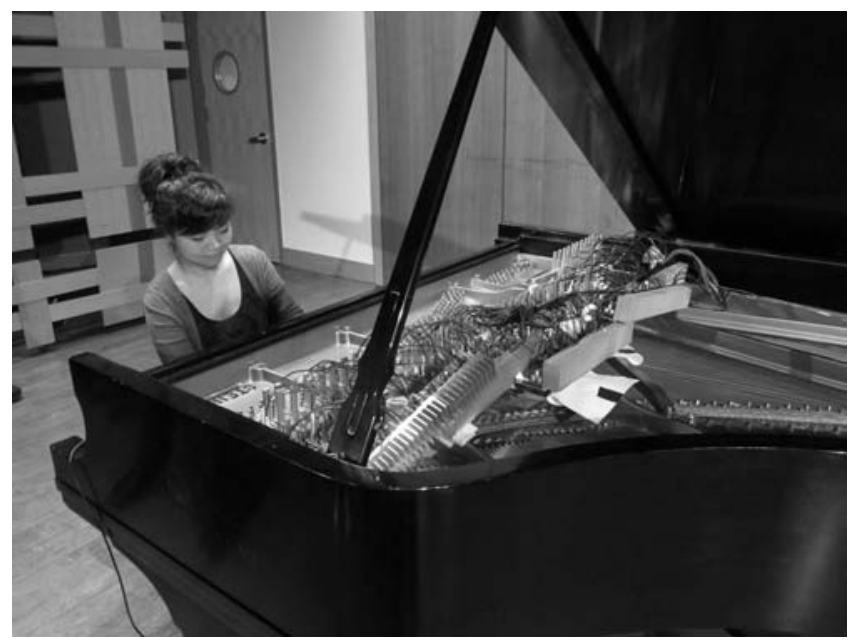

\section{Access Considerations}

The following sections discuss ongoing collaborations with composers and performers using the magnetic resonator piano (MRP). Like most augmented acoustic instruments, including a similar "electromagnetically prepared piano" (Berdahl, Backer, and Smith 2005), the MRP involves specialized hardware, so community-building is dependent on providing access to the equipment. Although a significant musical community can be built around a single physical instrument, DMIs that have achieved broad dissemination (e.g., the ReacTable [Jordà et al. 2007] and the Silent Drum [Oliver and Jenkins 2008]) often do so by replication of the hardware, either by the designer or by the community through publicly available plans. Hardware replication is also a goal of the MRP project, and the following discussion will consider both the experience of musicians and the practical requirements of providing access to a growing community of collaborators.

\section{The Magnetic Resonator Piano}

The magnetic resonator piano (MRP) is an electronic augmentation of the acoustic grand piano (see Figure 1). On the traditional piano, when a note is struck, it cannot be further shaped before its release. The MRP places 88 electromagnetic actuators
Figure 2. The magnetic resonator piano uses electromagnetic actuators to induce vibrations in the piano strings. The amplifiers are seen along the top of the photo and the actuators are seen above the strings.
Figure 3. An optical sensor (modified Moog Piano Bar) detects the continuous motion of each key.

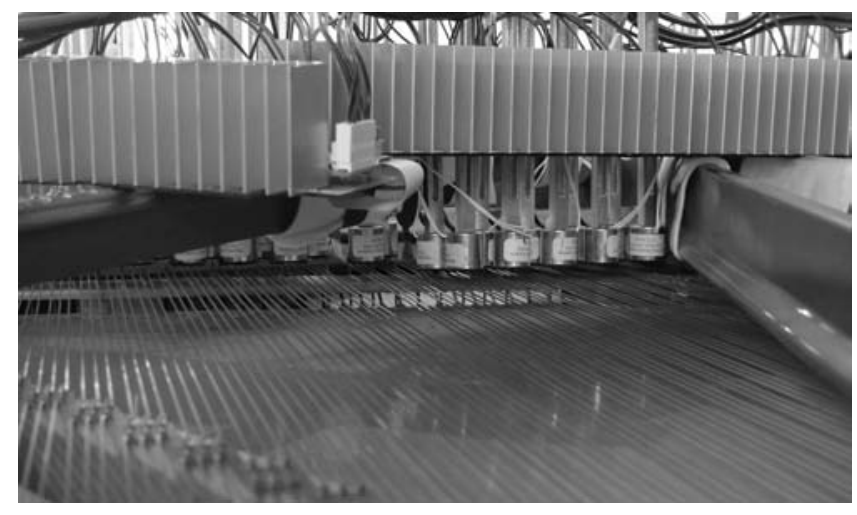

Figure 2

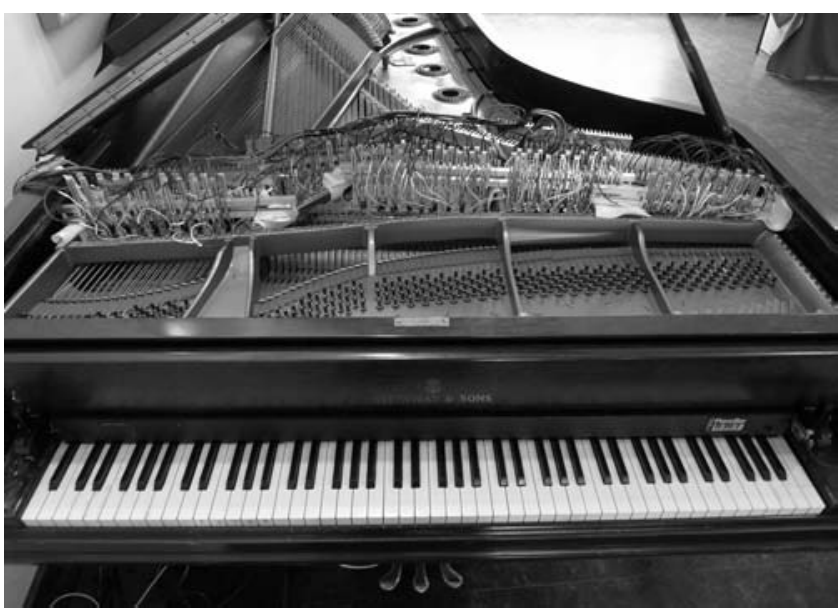

Figure 3

inside the piano that induce the strings to vibrate independently of the percussive hammer mechanism (see Figure 2). By varying the signals to the actuators, it is possible to continuously shape the amplitude, frequency, and timbre of every note. All sound is produced by the strings and soundboard without external speakers, maintaining the richness and resonance of the acoustic piano (McPherson 2010).

The MRP is played from the piano keyboard, which is equipped with an optical sensor strip measuring the continuous position of each key (see Figure 3). Traditional MIDI keyboards record only note onsets and releases; the MRP, by extracting features of key motion over time, can continuously control multiple independent parameters of each 
note (McPherson 2010). Though a computer is used to analyze key motion and produce signals for the actuators, the performer rarely if ever interacts with the computer directly. The user experience is similar to playing a traditional instrument: Physical gestures on the keyboard create sounds from within the piano. Nothing interferes with the hammer mechanism, so traditional and magnetic resonator sounds can be mixed in performance. The pianist can use a slow or shallow touch to produce resonator sounds with no hammer attack.

\section{Building on Traditional Performance Technique}

Performers spend decades developing and refining their instrumental technique. Most digital instruments, regardless of merit, do not receive the same amount of determined effort. For the MRP, as with most DMIs based on a traditional design, the need to attract new performers will naturally lead one to the community of musicians skilled in traditional practice. The response of these musicians will be significantly more positive when their existing skills can be deployed, repurposed, and extended.

Perry Cook observes that "some players have spare bandwidth, some do not" (Cook 2009). In designing (and redesigning) the MRP, the focus was on the word "spare." To be sure, not every player can maintain the entirety of their existing technique while also controlling new interface elements (one reason, perhaps, for limited adoption of augmented instruments so far). On the other hand, there are many aspects of pianists' existing performance gestures that can be fruitfully repurposed. The MRP extends traditional piano technique by examining continuous key motion, adding new means of control from the keyboard, including gradual key motion, pressure on depressed keys, taps on the key surfaces, and vibrato.

Two deliberate limitations maintain the playability of the MRP. First, nothing interferes with traditional hammer-actuated piano technique, ensuring that the years pianists spend learning their instrument can transfer to the MRP. Second, sound is only produced through interaction with the keyboard, so there is no risk of inadvertent body or arm movements triggering musical events. This strategy aligns with Steve Benford's argument that certain ancillary gestures, although important to the physical expression and theatricality of a performance, need not directly result in music production (Benford 2010). A related example in augmented instrument performance is pianist Sarah Nicolls's use of accelerometers: The sensors are placed in a hat rather than on the body, separating her deliberate engagement with the sensors from the ancillary motions of piano playing (Nicolls 2010).

\section{Compositional Practice}

The role of the composer in establishing a new instrument is arguably just as important as the role of the performer: A compelling repertoire of pieces will be a strong motivator for future performances. With notable exceptions (Ferguson and Wanderley 2010; Nicolls 2010), compositional practice is less studied than performance practice, but many of the same principles apply. Concert music composers spend many years honing the craft of writing for traditional instruments; writing for a completely new instrument without guidance from an established repertoire is a challenging task.

One author (McPherson) is a concert music composer. From personal experience, the ability to imagine and understand the sound of an instrument is a prerequisite to writing for it. The means by which the sound is controlled, including the interface and the gesture-sound mappings, are important in that they limit the space of possibilities, but the sound always comes first. A sound palette that is too wide or amorphous can be a powerful deterrent. The importance of constraints in guiding composition for DMIs has also been observed by other authors (Stewart 2009; Murray-Browne et al. 2011).

\section{Original Design Goals}

Original design and composition for the magnetic resonator piano followed Cook's model: The original MRP was built in 2009 in parallel with the composition of an extended work, Secrets of Antikythera. 
Design decisions, especially the performance interface and gesture-sound mappings, were informed by the needs of the piece, and the instrument's capabilities shaped the music. Shortly thereafter, author McPherson wrote another a second piece, d'Amore for viola and MRP. In place of a pianist, the violist's sound was pitch-tracked and used to selectively activate strings inside the piano, simulating the sympathetic vibrations of the Baroque viola d'amore. The hardware was fixed by the time of composition, but new mapping strategies were developed in parallel with the composition.

Affordances were a primary concern in the original MRP design. The instrument was intended to give the musician a specific set of capabilities:

1. Infinite sustain on a single note

2. Crescendos, including crescendos from silence

3. Harmonics on each piano string, in turn allowing microtonal intervals

4. Pitch bends

5. Timbre controllable independently of dynamic level

6. Continuous control of as many parameters as possible from the keyboard

7. Minimal interference with traditional piano technique

The first five capabilities are particularly relevant to compositional practice: They provide unique sonic resources while still relating to the traditional piano. As we will show in later sections, this combination of novel and familiar provided a point of departure for composers. The last two capabilities relate to performance practice, specifically, the goal of engaging traditional performance technique and making effective use of the player's bandwidth.

\section{Musician Feedback}

Secrets of Antikythera and d'Amore were performed in concert and later recorded for $C D$ release. The performers were all new to the MRP; Secrets of Antikythera in particular was performed by three pianists between 2009 and 2011. Working with performers on these pieces provided a valuable opportunity for feedback on the MRP's capabilities and usability.

Over the same time period, several interactive demonstrations of the MRP were given at universities, conservatories, music festivals, and open public events. Audiences included composers, performers, musicologists, engineers, and members of the public (children and adults). Demonstrations began with a brief presentation of the instrument's capabilities, followed by a period for hands-on exploration of the instrument. Some events also included a longer musical performance.

Finally, we had the opportunity to solicit individual informal feedback from many composers and performers. These activities collectively constituted the first step toward building an MRP community. They served to raise awareness of the instrument, to identify possible collaborators, and to gather suggestions for future design revisions. Notably, the future of the instrument was intended to be independent of these specific compositions, and collaborating with other composers to write new MRP music became a priority.

\section{Redesigning Constraints}

Demonstrations of the MRP generally highlighted its affordances. Our presentations showed specific, identifiable techniques the performer could use to continuously shape the sound of the piano. We found, however, that extended musical interactions followed Magnusson's model: When a new musician sat down at the instrument, the process usually turned to an exploration of the instrument's constraints. Starting from the demonstrated set of sounds, players often sought to establish a personal sense of what could be achieved and how. Most feedback focused on how constraints could be relaxed ("It would be great if the instrument could ..."). Over the course of two years, several recurring suggestions formed the basis for a redesign of the MRP.

\section{Relaxing Constraints: Frequent User Requests}

Musician feedback highlighted six areas where design adjustments would make the MRP more 
generically useful. Five areas are cases of relaxing musical constraints, the sixth purely practical. By addressing these requests, the new instrument acquired a superset of the old instrument's capabilities.

\section{Dynamic Range}

The MRP produces sound by electromagnetically resonating the piano strings. Though these "resonator" sounds can be quite intense, the original design could not match the volume of the traditional piano being played forte. Musicians frequently requested louder resonator sounds to match louder passages on the piano.

\section{Attack Time}

In contrast to the impulse-like actuation of a hammer strike, electromagnetically driven strings require many oscillations to reach their loudest sound. On the original MRP, resonator sounds could take anywhere from tens of milliseconds to several seconds to reach their final amplitude, with the bass register speaking especially slowly (McPherson 2010). Musicians found that the slow speaking time limited the instrument's utility in faster passages.

The MRP is intended to augment, not replace, the traditional piano. Because the hammers remain usable, we initially felt that slow resonator speaking time was not an issue. Many musicians wanted the ability to explore hammerless sounds of the instrument, however, even while using it in fast passages.

\section{Timbre}

Hammer-actuated piano notes contain dozens of harmonics, but the original MRP tones featured a strong fundamental with few overtones. As a result, piano and resonator timbres differed significantly.

Much of the difference is rooted in the mechanics of the instrument, and no electromagnetic system can precisely replicate the effect of the hammer striking the string. Nonetheless, musicians frequently requested resonator sounds closer in timbre to the hammer-actuated piano.

\section{Control and Mapping}

Nearly universally, musicians wanted the ability to control as many dimensions of the sound as possi-

ble. Polyphonic control of amplitude, frequency, and timbre (itself multidimensional) presents a significant challenge, not only in sensor design but also in mapping. Piano performance is already challenging, and requiring the performer to manipulate too many additional controls risks making the instrument unplayable.

Some musicians preferred to see additional sensors and interface elements beyond the keyboard, including foot pedals, extra knobs, or touch-screen interfaces. Others wanted as many dimensions as possible integrated into the piano keyboard itself. Because DMIs decouple user interface and sound production, it is possible to pursue both strategies in parallel. The two strategies represent fundamentally different visions of the instrument's identity, however, and we have relied on our original artistic goals in deciding which path to pursue. Our research thus far focuses on integrating additional control into the piano keyboard.

\section{Register}

The original design covered four octaves of strings centered around middle C (C4). Higher pitches could be played as harmonics on lower strings, but their volume was limited. Musicians requested a resonator system covering all 88 notes of the piano.

\section{Setup Time}

The MRP installs in any grand piano and removes cleanly. Setup in a new instrument typically takes two hours, most of which came from adjusting the actuator brackets to match the piano's string spacing. The original design could not be scaled up to 88 notes while still allowing installation in the time available before a concert. Broader adoption of the instrument thus required a more practical mechanical setup. 
Figure 4. New MRP design,

showing the rapid-adjust

bracket inside the piano.

\section{Other Suggestions}

Some suggestions, though compelling, were impractical to implement. Some musicians requested that the resonators actively damp the strings as well as excite them. Active string damping is possible (Berdahl, Niemeyer, and Smith 2008), but its implementation on the grand piano is extremely challenging, requiring independent actuation of the three strings within a single note, individual pickups collocated with the actuators, and near-zero latency signal processing.

Other suggestions were not pursued because we felt they ran counter to the instrument's character. The primary such case was the creation of a sequencer to let the pianist trigger groups of sounds rather than individual notes (form-level rather than note-level or timbre-level control [Wanderley and Orio 2002]). We did use sequencing techniques to a limited extent within particular pieces, but in the general case we preferred to maintain the MRP as an instrument in the traditional sense.

\section{Hardware Changes}

Bringing a DMI to a larger musical community frequently entails design changes, especially to the mapping strategies between gesture and sound. For the MRP, relaxing constraints identified by musicians required a fundamental redesign of the electromagnetic actuation hardware.

\section{Amplifier Design}

Several constraints resulted from the amplifiers that drive the actuators:

1. Volume was limited by total amplifier power.

2. Attack time was limited partly by amplifier power, and partly by signal pre-processing steps between computer and amplifiers.

3. Timbre, especially in the case of bright timbres, was limited by the high-frequency performance of the amplifiers and actuators. This in turn was limited by the slew rate (maximum slope) of the actuator current, which was ultimately dependent on the voltage swing of the amplifier.

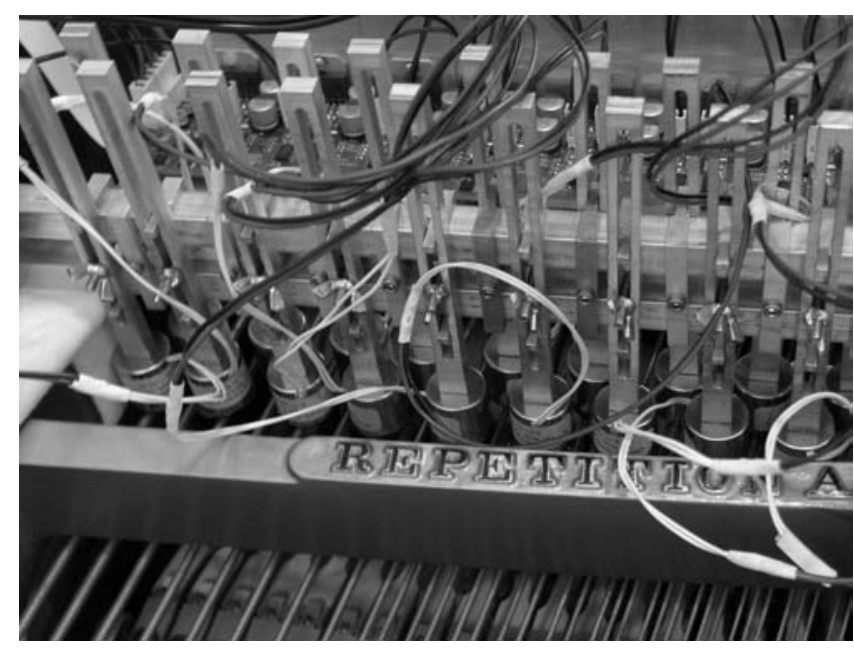

The original amplifier design can be found in McPherson (2010). The new design produces enough power that actuator heating, not amplifier performance, becomes the limiting factor, and it doubles the output voltage swing. A technical analysis of the new design can be found in McPherson (2012); this article will instead maintain focus on the instrument's musical implications.

\section{Actuator Design}

The original MRP actuators were wound by hand, a tedious process. The new MRP uses machinewound actuators, which provide better performance over all 88 notes. Because the new design is machine-producible, it allows multiple copies of the instrument to be built. As yet, only a single MRP has been produced on the new design, but replicability and access are important considerations in DMI community-building, especially for composers interested in having their pieces played repeatedly.

We also developed a new actuator bracket design (see Figure 4) that allows each actuator to be moved in two dimensions and locked with a single wingnut. The new bracket can be machine-produced and helps address the setup time constraint. 


\section{Mapping for Performance}

Redesign of the MRP hardware was necessary to relax constraints on volume, attack time, and timbre. Addressing musician requests on controlling the resonator sounds focused on refining the mapping between keyboard and electromagnet behavior. Albert Einstein is often quoted (apocryphally) as saying "everything should be made as simple as possible, but not simpler." Similarly, based on Cook's bandwidth observation, we can state our mapping principle as: "Put as much control as possible in the hands of the performer, but no more." The MRP will be played primarily by pianists with a highly developed gestural vocabulary. Most potential performers felt that extra dimensions of control would give them more expressive freedom, but existing piano technique leaves relatively few spare motions. Our mappings sought to find means of control from within traditional technique while minimizing the number of new interface elements.

\section{Augmented Keyboard Interface}

When Secrets of Antikythera was composed in 2009, the MRP was played from two keyboards: the piano keyboard, using a Moog Piano Bar (Computer Music Journal 2005), and an auxiliary MIDI keyboard atop the piano. We found that MIDI note onsets and releases were inadequate for continuously controlling multiple dimensions of a note.

We thus modified the Piano Bar to extract continuous key position, measured at 600 samples per second per key. Continuous key position enabled a variety of extended techniques: gradual and partial key presses, taps and sweeps on the key surfaces, pressure into the key-bed, and vibrato gestures. It also allowed a detailed investigation of piano "touch" that showed that a single press gesture could be independently varied in multiple dimensions beyond MIDI velocity (McPherson and Kim 2011).

\section{One Mapping or Many?}

Multiple continuous features could be extracted from key motion, and multiple parameters of each
Figure 5. Two-layer

mapping from key motion

to actuator parameters.

The second layer is

dynamically adjustable;

the standardized mapping

is shown in solid black

lines.

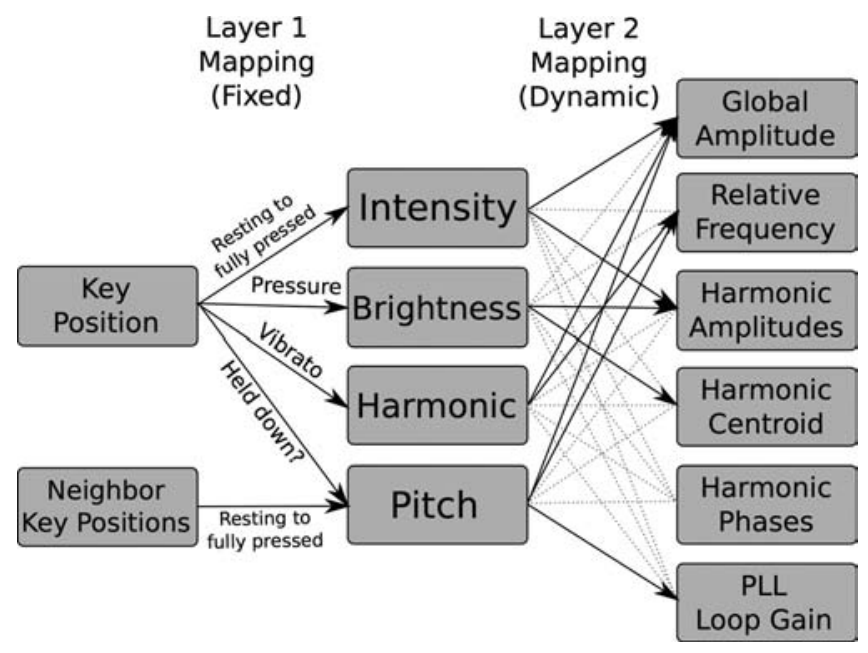

electromagnet can be controlled. The challenge is how the two parameter spaces should relate. In working with musicians, it became clear that the needs of composers and improvisers could be quite different, and consequently, no one mapping strategy could serve all cases. For the improviser, as many sounds as possible should be available at the fingertips; for notated music, the piece can determine what sounds need to be available at any given time. For pieces that need only a few sounds, limiting the dimensions of control reduces the complexity for the performer, potentially resulting in a better performance.

\section{Toward a Standardized Mapping}

Moving from a composer-specific instrument to a more generically usable one required a mapping suited to a wide variety of musical situations. This standardized mapping will be what a new performer first encounters, and we designed it to be one improvising musicians could use. Although it is not possible to simultaneously, independently control every sonic parameter, we aimed to provide as many dimensions as possible in a way that is repeatable and reliable and that encourages exploration. The standardized mapping uses a two-level strategy (see Figure 5): 
1. Features of key motion are mapped to four intermediate parameters: intensity, brightness, harmonic, and pitch. The parameters, whose meanings are explained in the following, are quasi-independent in that not all combinations are possible but none is a linear combination of the others.

2. Intermediate parameters are mapped to actuator behavior, controlling amplitude, frequency (relative to string fundamental), and waveform (combinations of harmonics, affecting amplitudes, phases, and centroid in Figure 5).

Layer 1 is fixed in code; Layer 2 is can be dynamically adjusted based on an XML parameter file. Mappings in Layer 2 are one-to-many and can be linear or logarithmic, so the entire system is a many-to-many mapping between features of key motion and actuator parameters. Both layers relate specifically to actuator behavior; because no mechanical alterations are made to the piano, hammer strikes (when present) maintain their normal function.

In Layer 1, raw key position from top to bottom controls intensity. When the key reaches the keybed, further pressure controls brightness. (Because felt separates the key from the key-bed, pressure produces a slight deviation in position.) A vibrato gesture on a resting key, produced by light taps or gripping key between thumb and forefinger, controls harmonic, whose value increases linearly with time at a rate proportional to the vibrato speed. Pitch is controlled by a combination of two adjacent keys: When one "center" key is held, partial presses on neighboring white keys bend the pitch of the center key up or down. This mapping temporarily overrides the intensity parameter on the adjacent key.

With the exception of raw key position, none of the techniques just mentioned (pressure, vibrato, partial presses) appear in traditional piano performance. At the same time, the gestures can be easily learned and executed. The names of the parameters reflect their usual function, but they can be freely mapped to any sonic parameters. In the standardized mapping, intensity controls (logarithmic) amplitude and waveform, producing louder, brighter tones for deeper key presses. Brightness further controls waveform, pushing the centroid of the spectrum higher. Pitch maps logarithmically to frequency (a semitone in either direction) as well as to amplitude. Harmonic maps to frequency as an integer multiple of the fundamental and amplitude. Both frequencyrelated effects increase the amplitude to compensate for the string's reduced response away from its fundamental frequency. An additional parameter, loop gain, is needed to bend pitches away from the string's natural frequency (McPherson 2010).

\section{Real-Time Mapping Adjustment}

The standardized mapping allows real-time exploration of volume, pitch, and certain aspects of timbre. It represents a compromise between detail of control and playability, and thus certain sounds are not easily accessible. Limitations include a restricted range of timbre, inability to polyphonically select different harmonics on different notes, and relatively constant volume when keys are held all the way down (as they are in traditional piano playing, regardless of attack velocity).

The XML file defining Layer 2 can be reconfigured for every piece. It can also define multiple mappings, selectable with MIDI Program Change or Open Sound Control (OSC) messages. Each mapping program can define behavior on a key-by-key basisfor example, allowing only a certain range of pitches to have the resonator added. Global resonator volume can also be externally set by MIDI or OSC. Mapping changes lack the expressive immediacy of keyboard control, but they allow access to a wider range of sounds and are especially useful in notated compositions. The next section shows how they were used in specific pieces.

\section{Composer Collaboration}

We undertook a yearlong project in which six composers from Philadelphia, Pennsylvania, and Princeton, New Jersey, were invited to write new pieces for the MRP. The composers were David Carpenter, William Derganc, Daniel Fox, Daniel 
Shapiro, Jeffrey Snyder, and Tony Solitro. At the time of composition, the group included one finalyear undergraduate, three graduate students, and two post-PhD professional musicians. Each participant had previously seen the instrument at a concert performance or interactive demonstration, but none had been involved in its development. One composer (Snyder) worked primarily with electronic and novel instruments; the other five had previously composed primarily for acoustic instruments, though two (Solitro and Derganc) had incorporated electronics in previous pieces.

At the start of the project in early 2011, we decided to present two performances of the new pieces. Particularly with new or unusual instruments, multiple performances better justify the time composers spend creating new works. Four composers (Fox, Shapiro, Snyder, and Solitro) chose to write solo pieces for MRP; one composer (Derganc) wrote for violin and MRP; and one composer (Carpenter) wrote for baritone voice and MRP. Aside from the MRP, no other electronic elements were used in any piece.

\section{Working Methods}

Access to the instrument was a crucial component of the composition process, and this was made easier by the fact that all composers were local to Philadelphia, where the project was carried out. Most composers' first direct contact with the instrument was at a group demonstration session (described earlier). Each composer subsequently came to the Music and Entertainment Technology Laboratory at Drexel University, where the MRP was set up, to work individually with the instrument. Individual sessions with the instrument included guided explorations (engaging with the instrument's intended affordances) and unstructured improvisation (exploring constraints).

Composers took varying approaches to the writing process. One composed mostly at the MRP; three others composed mostly away from the MRP but at a keyboard; two more indicated they did not use a keyboard at all. In a follow-up survey, one composer explained:
[W]hile the entirety of my work was physically written away from the instrument, the bulk of the compositional process was shaped by several meetings well in advance (greater than six months) of the work's completion. I rarely work at the intended instrument(s) for which I compose, as it is usually not pragmatic; however, in this case, I (somewhat subconsciously) believed it might be a hindrance to my imagination if I were to content myself with producing the sounds I could produce at the instrument, thereby letting my own technical limitations dictate the scope of the work's sound-world.

In general, even the composers who worked away from the instrument indicated that the original meetings shaped their ideas or served as an opportunity to test sounds before including them in the piece. One composer wrote an initial version of the piece for piano and external electronics (Max/MSP), with the idea of later transferring it to MRP. He felt that this process "freed me to imagine the sounds I wanted, and then to find an effective realization after the fact."

Sometimes, either as a result of experimentation or as an idea developed during composition, composers would request sounds that were not easily achievable with the standard mapping between keyboard and actuators. We encouraged the composers not to feel limited by the default set of mappings, and in this case we would work to develop custom mappings to allow the intended sound to be performed. Composer Shapiro, exploring the pitch bends that are played with two adjacent keys in the standard mapping, was drawn to the sound of the resonator when bent slightly below the natural pitch of the string. He requested a mapping that would produce this out-of-tune sound on every note by default. This was achieved by adjusting the relative center frequency in the second-stage mapping layer described in the previous section; the resulting mapping was used in the second movement of Shapiro's piece (Sound Examples 1 and 2). [Editor's note: This article's sound examples are posted online at www.mitpressjournals.org/toc/comj/36/4 and will 
also appear on the 2013 Computer Music Journal Sound and Video Anthology DVD.]

\section{Rehearsal and Performance}

Once the draft scores were complete, we worked with the composers and performers to finalize how each piece would be performed. This step included implementing piece-specific mappings, getting feedback from performers on the most natural way to perform certain techniques, and working with the composer to develop a consistent notation for any novel effects.

Performers for the project included four pianists, a violinist, and a baritone vocalist. Before the project began, none of the pianists had previously played the MRP. Two pianists worked with the composers during the composition process, with pianist Feifei Zhang taking a role in three pieces over the months leading up to the concert; the other three pianists joined the project during the final rehearsals. The rehearsal process served to familiarize the performers with the MRP and to provide feedback to the composers, who sometimes made changes to the score in response to early rehearsals.

Two performances were given in December 2011, one at Drexel University and one at Temple University. The resonator system was installed in a Steinway D grand piano in both cases, though a different instrument at each venue. Because the response of the resonator system in the concert pianos differed from the 5-foot-long Knabe baby grand used for early rehearsals, an extended dress rehearsal before the first concert gave composers a chance to make last-minute adjustments.

During the performances, author McPherson sat in the front row of the auditorium, using an iPod touch to dynamically adjust mappings and overall volume via OSC. Separate mappings were loaded for each piece, and for some pieces, mappings and volume were adjusted several times within the piece. Though some of these controls could have been given to the pianist, we decided that it was better to minimize the number of new techniques the pianist needed to learn, especially for players who joined the project in the final rehearsals. In all cases, primary expressive control remained with the performer, as there were few scripted or sequenced actions in any piece.

\section{Results and Observations}

All six composers who started the project completed pieces, and all pieces were successfully presented in concert. In the week following the concert, composers were given a written questionnaire covering their experiences on the project and their suggestions for improvement. Based on this questionnaire, the completed scores, and observations of the composition process, several interesting trends emerged.

\section{Notation}

Ferguson and Wanderley argue that the ability to consistently reproduce a performance from notation is a primary metric of success for DMIs (Ferguson and Wanderley 2010). We decided early in the project not to attempt to prescribe a uniform set of notational conventions. The capabilities of the instrument had evolved since Secrets of Antikythera was written in 2009, and it was also expected that each composer would imagine different sounds and techniques. With guidance from the authors, each composer was encouraged to develop a personal notation system, with the idea that similarities in approaches could guide eventual standardization.

Notation strategies could be classified according to whether they noted the sound of a gesture or its means of execution. In the former category, all composers used standard hairpin notation for dynamics: Crescendos were notated as they would be on any other acoustic instrument. Likewise, pitch bends were mostly notated as they sounded rather than how they were played (a gesture involving two adjacent keys).

In the standard MRP mapping, a resonator sounds for as long as its key is held down. If the key is released with the damper pedal depressed, the note will then follow the natural decay of the piano. By contrast, in traditional piano technique the 
Figure 6. Excerpt from Spectra of Morning by Tony Solitro. Long notes, as well as notes followed by black horizontal bars, are played with organ sustain. Staves below the piano indicate audio

played through the piano

strings. (Sound Example 3;

excerpt starts at 0:24.)

Copyright (C) 2011 by

Tony Solitro

$\sim$ tonysolitro.com.
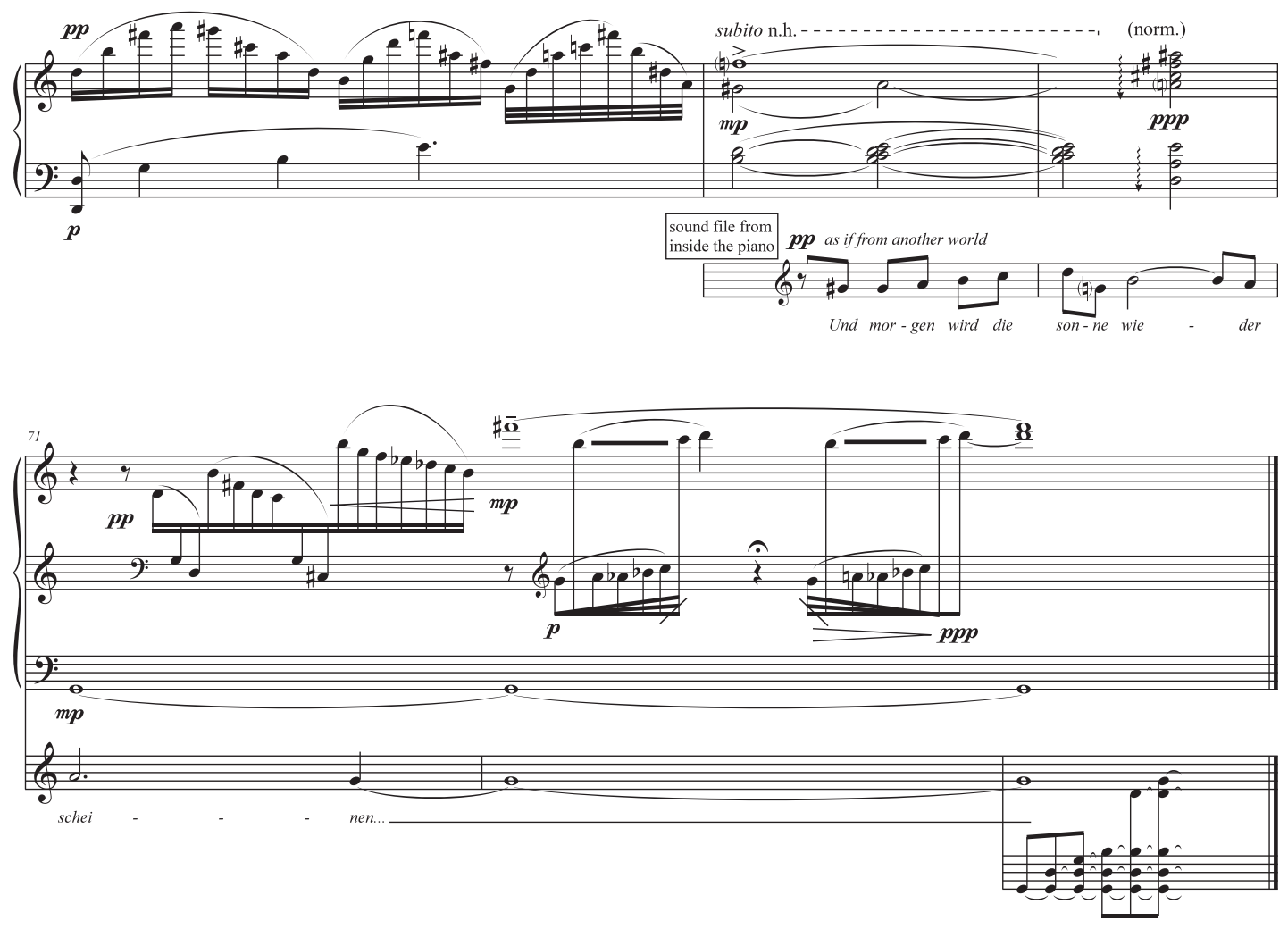

pianist can often choose whether to sustain notes by holding the keys or using the pedal without a large perceptual difference in sound. We adopted the term "organ sustain" to refer to notes sustained with the resonator and "piano sustain" for notes allowed to decay with the pedal down; some pianists found adjusting to this distinction one of the more difficult aspects of MRP technique.

In Tony Solitro's Spectra of Morning (see Figure 6; Sound Example 3), the long notes are held with organ sustain. The effect is especially significant on the bass pedal tones, which can be held for multiple bars at a time. Solitro also uses solid black lines to indicate shorter notes within the texture that use resonator to persist beyond their notated length. The remaining notes are played with piano sustain (the damper pedal held but no resonator added).

A related distinction concerned notes played with resonator but without hammer. Most composers used standard notation to indicate notes played with hammer, using the phrases "no hammer", "n.h." or diamond noteheads to indicate resonator-only passages. David Carpenter's Job took the opposite approach (see Figure 7; Sound Example 4). By default, notes are played with resonator only and held with organ sustain. Triangular noteheads indicate pitches played with both hammer and resonator.

Effects based on harmonics or timbre had fewer traditional notation strategies to draw on. In these cases, notation focused primarily on how the effects were executed with a secondary emphasis on showing the sonic result. Daniel Shapiro's The Masons of Heidelberg (see Figure 8; Sound Examples 1 and 2) used harmonic glissandos, pitch bends, and timbre changes within notes. In the standard mapping, upward harmonic glissandos are produced by gently vibrating the key between thumb and forefinger; in consultation with the authors, Shapiro used a diagonal wavy line (m. 30) which signifies a combination of the vibrato gesture 
Figure 7. Excerpt from Job by David Carpenter. Standard noteheads are played with resonator only, triangular noteheads (m. 39) with resonator and hammer. (Sound Example 4.) Copyright (C) 2011 by David Carpenter. All rights reserved.
Figure 8. Excerpt from The Masons of Heidelberg (movt. I) by Daniel Shapiro. Extended techniques include harmonic glissandos (m. 30), aftertouch timbre modification, and pitch bends (m. 34). Sound Example 1 is from earlier in this movement; Sound Example 2 is from movement II.
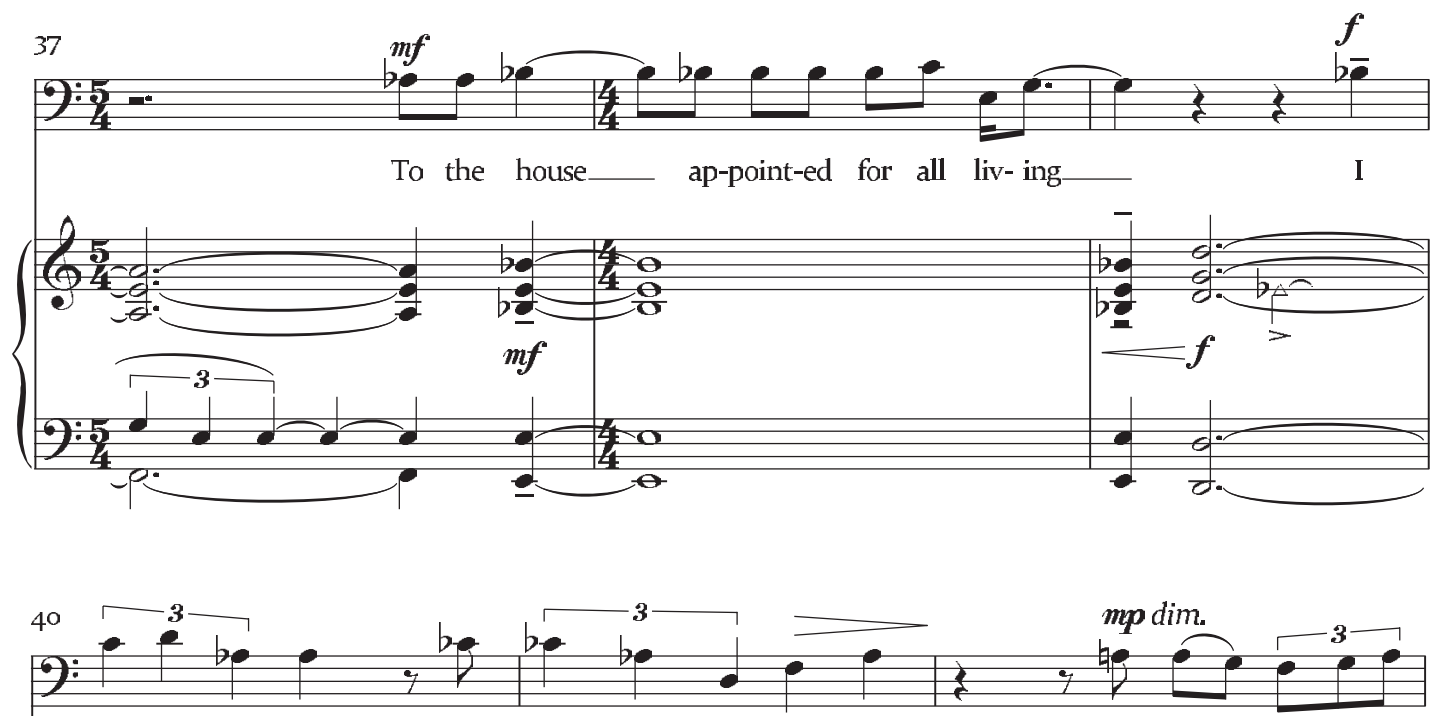
cry un - to thee, and thou dost not hear me. Mine eye - pour-ethout

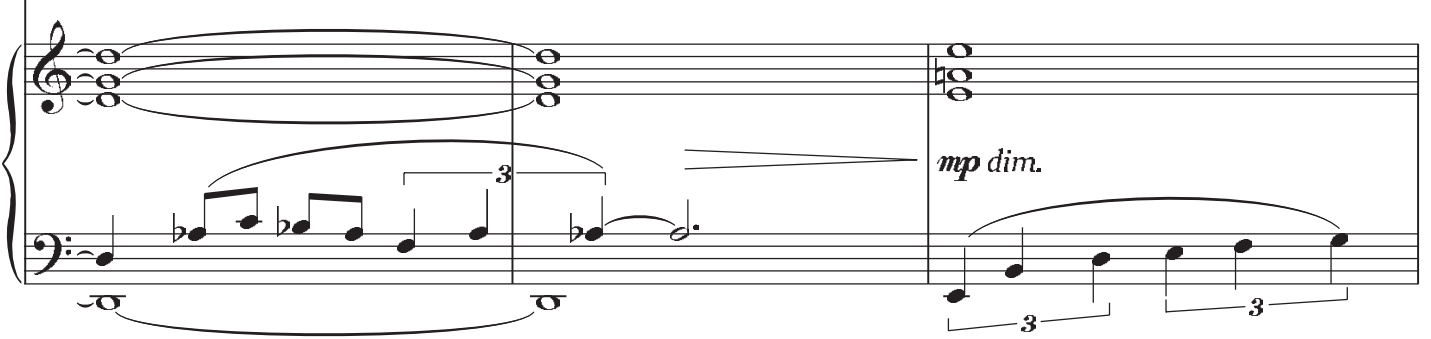

Figure 7

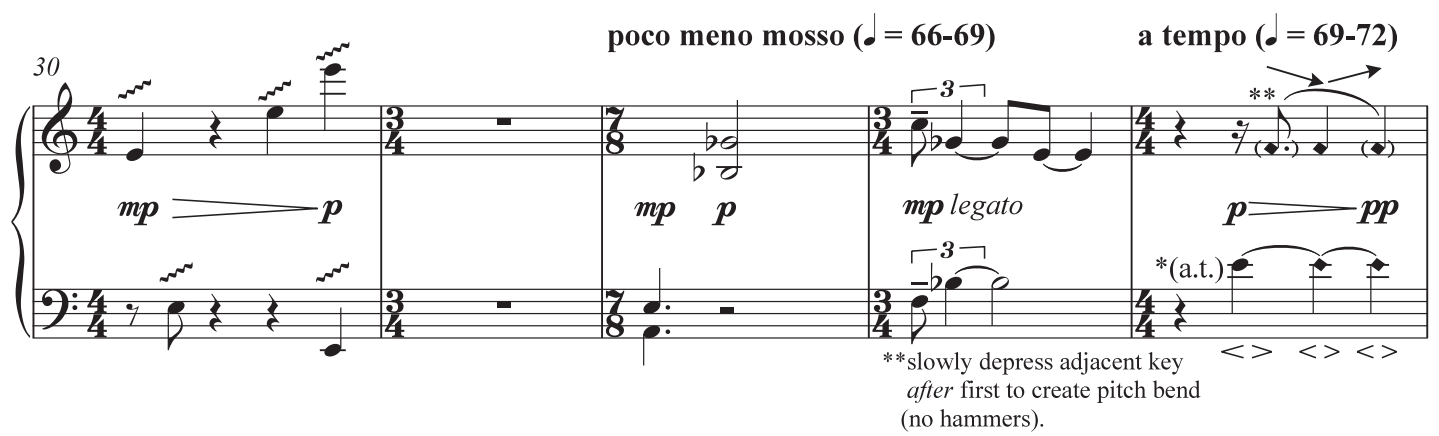

Figure 8

and the resulting sweep up in pitch. In the last measure of Figure 8, two extended techniques are used together. Aftertouch (key pressure) is used to create a brighter timbre; Shapiro notates this using hairpins and the marking "a.t." Simultaneously, a pitch bend pulls the pitch of the E upward. The pitch bend is executed by lightly pressing the neighboring key $(\mathrm{F})$; Shapiro's notation focused on the method of 
execution by showing the direction of key motion rather than the direction of pitch bend. In all scores, unfamiliar notations are explained in a preface or appendix.

\section{Relationship to the Traditional Piano}

Augmented instruments provide a challenge for composers: To what extent should the long tradition of the underlying acoustic instrument guide the composition? The composers' approaches can be divided into three categories: piano-driven, resonatordriven, and oppositional. The piano-driven approach treats the MRP as an extension of the acoustic piano, using traditional piano technique as the foundation of the piece. Composer Fox (Sound Example 5) wrote: "I tried to use the resonator sounds as a continuous outgrowth of the piano sounds. I used the resonator for organ-like percussion-less attacks, for sustain, and for new tone qualities." Derganc (Sound Example 6) wrote: "I used the MRP in a very limited way. ... I wanted a very even mixture of piano and MRP in my piece." Both pieces are driven primarily by traditional (hammer-actuated) piano technique with resonator used to color and extend the piano sound. For Solitro, too, the resonator acted as an outgrowth of the traditional piano: "For my piece, the resonator sounds often sustain notes and chords in ways that would otherwise be impossible. [MRP pedal tones] provide a harmonic foundation, while the piano plays traditionally sounding ornate and fantasy-like passage-work against the pedal."

Other composers concentrated on resonator sounds while pushing traditional piano technique to the background. Carpenter primarily used sounds with no hammer attack, eliciting an organ-like quality from the MRP. Shapiro also challenges the primacy of the piano: "I don't hear much 'traditional' piano in my work, as I don't hear the MRP as an augmented piano. It is simply a new instrument that bears a relationship to the piano, much the same as the harpsichord bears a relation to the piano, in terms of mechanism alone."

Jeffrey Snyder's approach to the MRP is best described as oppositional. Snyder writes: "In my piece, the resonator sounds are generally microtonal ... by contrast, all the traditional piano writing uses equal-temperament (by necessity) so the combination of resonator and traditional piano represent a sort of struggle and interaction between the tuning systems." Snyder's Fantasy is played from two keyboards, with the top keyboard controlling resonators alone and the bottom keyboard hammers alone, so there is no overlap in the sound sets.

\section{Mapping and Control}

Each piece used a different set of mappings, sometimes multiple mappings within a single piece. Mapping changes were primarily used to change timbre and which notes had the resonator added, a role analogous to stops on an organ. Carpenter, Derganc, Fox, and Shapiro used variations on the standard mapping, which uses continuous key position to shape each note. In contrast, Solitro used standard MIDI (onset and release) from the piano keyboard, which proved more reliable for sustain effects where continuous shaping was not required. Snyder used MIDI from an auxiliary keyboard, with the lowest octave of keys reserved for changing mappings. In Figure 9, notes C2-B2 change the harmonic (1-12, respectively) of the following notes. Notes C3 and above play the previously selected harmonic on the strings two octaves below notated; for example, the C\# at the beginning of m. 3 will play the third harmonic (G\#4) of the strings for the note C\#3. The sounding pitches will thus be markedly different from the notated pitches.

Tony Solitro's Spectra of Morning contained several short passages in which a sequence of notes or a pre-recorded sound was played through the resonator system. In Figure 6, a recording of a vocalist is played into several resonators $(\mathrm{G} 3, \mathrm{G} \# 3$, A3, B3, C4, and D4, one octave below each pitch in the excerpt) to produce a ghostly, reverberant shadow of the original sound from within the piano. These effects were triggered externally (by the author acting as patch-changer) and represent one of the few cases that the pianist did not have note-level control over the result. 
Figure 9. Excerpt from Fantasy by Jeffrey Snyder. (a) Notes in lowest octave select harmonics for following notes (C2 = 1 to B2 = 12). (b) "pno." and "e.k." indicate piano keyboard and electronic MIDI keyboard, respectively. (Sound Examples 7 and 8 [starting at $0: 14]$.)

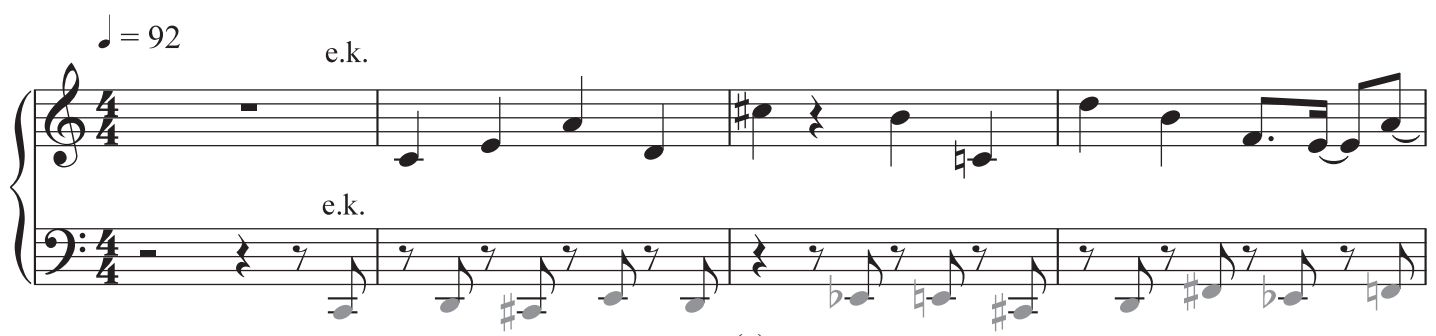

(a)

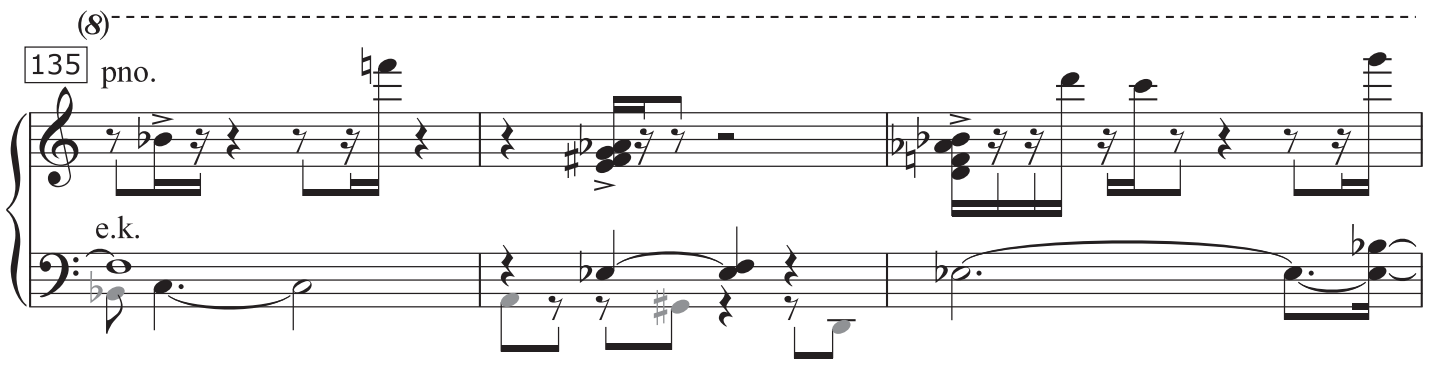

(b)

\section{Composer Feedback}

Feedback from composers was very positive. Most composers indicated that the results were close to their expectations. One composer wrote that his piece "turned out very true to my expectations, and sounded better on many things than I had imagined. Especially upon the switch to the Steinway D concert grand for the performance, many of the subtle details I had written into resonator-intensive sections of the piece came out beautifully."

We asked each composer to suggest improvements to the instrument. One requested a GUI to allow easier exploration of new timbres. Two composers found the piano dampers limiting: The damper must be lifted for a resonator note to sound, requiring either the key or the pedal to be held. The composers suggested that a MIDI-enabled piano might allow dampers to be lifted automatically as required.

Several suggestions echoed feedback we had received about the first revision of the instrument. Two composers requested resonator tones that speak more quickly, allowing faster passages without hammers. One composer requested resonator timbres that more closely match the traditional piano. These comments suggest that the design revisions were targeted to relevant areas, but only partly satisfied the composers' requests. Finally, several composers wanted greater control from the keyboard over resonator dynamics. Alternative mappings for volume will be explored that don't require the performer to partially press keys to achieve softer sounds. Like earlier feedback, composer comments focused on ways to relax constraints of the current instrument.

\section{Conclusions}

Establishing a continuing musical role for a digital musical instrument is a challenge. We have presented a case study in musical community-building using the magnetic resonator piano. Initial musician feedback guided a complete revision of the hardware system with a focus on relaxing constraints. A collaborative project with six composers and four pianists resulted in the performance of six new pieces for MRP. We intend to present further performances of this repertoire and establish more collaborations with performers and composers. Particular priority will be placed on working with improvising 
performers, who may take a different approach to the instrument than composers of notated music.

\section{Recommendations for DMI Designers}

Based on our experience with the MRP, we offer the following suggestions to instrument designers seeking to establish their instrument in a broader community:

1. Design for the first performance; then iterate. The instrument and the piece may initially be linked, but they are not equivalent; it is not possible to build a community around a piece. Once the first performances are finished, the designer should focus on how to let other musicians take personal artistic ownership of the sounds and techniques, a process which will likely focus on relaxing constraints. It is not necessary to involve a large number of musicians in the first design, but feedback is important to the revision process.

2. Demonstrate uniqueness, but connect to familiar models. Potential users of the instrument should have a compelling reason to use the new design rather than existing instruments. Every professional musician has spent many years acquiring a specific skill set, however, and where the techniques and sounds of a new instrument can be connected to existing experience, greater adoption and better performances are likely to result.

3. Sell to the audience; follow up with the performer or composer. The first encounter a potential collaborator will have is likely to be as an audience member. A convincing performance from the audience perspective will help convince performers and composers to explore the instrument in more detail.

4. Provide access. Access is important during the artistic creation process: Composers and performers will need regular interaction with the instrument to produce pieces. Even where it is possible to make multiple copies of an instrument, access to the designer is also important for answering questions and suggesting areas of exploration. Access is also important after the collaboration is finished. Composers want to know their pieces will have a continuing performance life, and performers will want to spend their time acquiring skills that can be reused. A strong commitment to pursuing the instrument beyond the duration of a research project can itself be a motivator to acquiring and maintaining a broad community of collaborators.

\section{Acknowledgments}

Many thanks to the composers involved in the project and to all the performers: Feifei Zhang, Katya Popova, Eric Wubbels, Matthew Bengtson, Lawrence Indik, and Noco Kawamura. Thanks also to members of the Drexel University Music Entertainment Technology Lab and the Temple University Music Department for making the concerts possible. This article is based upon work supported by the National Science Foundation under grant no. 0937060 to the Computing Research Association for the CIFellows Project.

\section{References}

Benford, S. 2010. "Performing Musical Interaction: Lessons from the Study of Extended Theatrical Performances." Computer Music Journal 34(4):49-61.

Berdahl, E., S. Backer, and J. O. Smith. 2005. "If I Had a Hammer: Design and Theory of an ElectromagneticallyPrepared Piano." In Proceedings of the International Computer Music Conference, pp. 81-84.

Berdahl, E., G. Niemeyer, and J. O. Smith. 2008. "Feedback Control of Acoustic Musical Instruments." Technical Report STAN-M-120. Palo Alto, California: Stanford University.

Computer Music Journal. 2005. "Products of Interest." Computer Music Journal 29(1):104-113.

Cook, P. 2009. "Re-Designing Principles for Computer Music Controllers: A Case Study of SqueezeVox Maggie." In Proceedings of the Ninth International 
Conference on New Interfaces for Musical Expression, pp. 218-221.

Ferguson, S., and M. M. Wanderley. 2010. "The McGill Digital Orchestra: An Interdisciplinary Project on Digital Musical Instruments." Journal of Interdisciplinary Music Studies 4(2):17-35.

Gurevich, M., and A. Fyans. 2011. "Digital Musical Interactions: Performer-System Relationships and Their Perception by Spectators." Organised Sound 16(2):166-175.

Gurevich, M., P. Stapleton, and A. Marquez-Borbon. 2010. "Style and Constraint in Electronic Musical Instruments." In Proceedings of the Tenth International Conference on New Interfaces for Musical Expression, pp. 106-111.

Jordà, S. 2004. "Instruments and Players: Some Thoughts on Digital Lutherie." Journal of New Music Research 33(3):321-341.

Jordà, S., et al. 2007. "The reacTable: Exploring the Synergy Between Live Music Performance and Tabletop Tangible Interfaces." In Proceedings of the First International Conference on Tangible and Embedded Interaction, pp. 139-146.

Magnusson, T. 2010. "Designing Constraints: Composing and Performing with Digital Musical Systems." Computer Music Journal 34(4):62-73.

McPherson, A. 2010. "The Magnetic Resonator Piano: Electronic Augmentation of an Acoustic Musical Instrument." Journal of New Music Research 39(3):189202.

McPherson, A. 2012. "Techniques and Circuits for Electromagnetic Instrument Actuation." In Proceedings of the Twelfth International Conference on New Interfaces for Musical Expression (pages unnumbered).

McPherson, A., and Y. Kim. 2011. "Multidimensional Gesture Sensing at the Piano Keyboard." In Proceedings of the 29th ACM Conference on Human Factors in Computing Systems, pp. 2789-2798.

Murray-Browne, T., et al. 2011. "The Medium is the Message: Composing Instruments and Performing
Mappings." In Proceedings of the Eleventh International Conference on New Interfaces for Musical Expression, pp. 56-59.

New York Times. 2011. Obituary: Steven P. Jobs. http://www.nytimes.com/2011/10/06/business/ steve-jobs-of-apple-dies-at-56.html, 5 October.

Newton, D., and M. T. Marshall. 2011. "Examining How Musicians Create Augmented Musical Instruments." In Proceedings of the Eleventh International Conference on New Interfaces for Musical Expression, pp. 155-160.

Nicolls, S. 2010. "Seeking Out the Spaces Between: Using Improvisation in Collaborative Composition with Interactive Technology." Leonardo Music Journal 20:47-55.

Oliver, J., and M. Jenkins. 2008. "The Silent Drum Controller: A New Percussive Gestural Interface." In Proceedings of the International Computer Music Conference, pp. 651-654.

O'Modhrain, S. 2011. "A Framework for the Evaluation of Digital Musical Instruments." Computer Music Journal 35(1):28-42.

Paine, G. 2009. "Towards Unified Design Guidelines for New Interfaces for Musical Expression." Organised Sound 14(2):142-155.

Rasamimanana, N. 2012. "Towards a Conceptual Framework for Exploring and Modelling Expressive Musical Gestures." Journal of New Music Research 41(1):3-12.

Redström, J. 2006. "Towards User Design? On the Shift from Object to User as the Subject of Design." Design Studies 27(2):123-139.

Schloss, W. A. 2002. "Using Contemporary Technology in Live Performance: The Dilemma of the Performer." Journal of New Music Research 32(3):239-242.

Stewart, D. A. 2009. "Digital Musical Instrument Composition: Limits and Constraints." In Proceedings of the Electroacoustic Music Studies Network Conference (pages unnumbered).

Wanderley, M. M., and N. Orio. 2002. "Evaluation of Input Devices for Musical Expression: Borrowing Tools from HCI." Computer Music Journal 26(3):62-76. 